\title{
Property-Based Taming of Lying Mobile Nodes
}

\author{
Mark Manulis* Ahmad-Reza Sadeghi \\ Horst-Görtz Institute, Ruhr-University of Bochum \\ D-44801 Bochum, Germany \\ mark.manulis@rub.de, sadeghi@crypto.rub.de
}

\begin{abstract}
Intelligent security protocols can verify whether the involved principals have properties that are defined based on certain functional and security policies. The property we focus on is the performance of mobile devices participating in a security protocol. In this context, the protocol should distribute the computation, communication and storage costs fairly among all devices. However, the protocol should foresee against cheating participants who may lie about their properties to gain advantage.
\end{abstract}

\section{Introduction}

Many security applications in practice are based on interactive computation among mistrusting parties. Basically, each party inputs own secrets to the protocol and receives the (secret) output at the end. The protocol has to guarantee the security requirements which can vary depending on the application. In this context it is important to ensure the trustworthiness of the involved principals with respect to certain (security) policies. More concretely, we want to enforce that a mobile device taking part in the security protocol has the desired property, otherwise the principal should not be granted access to the output of the protocol.

In this paper, we consider a group key agreement for heterogeneous mobile ad-hoc groups [6]. These are groups where principals are equipped with different kinds of mobile devices such as cell phones, PDAs, laptops. Obviously, performance capabilities vary among devices due to different hardware and software configurations. Taking this into account we are interested in a fair distribution of the computational load among the devices. The protocol in [6] distributes computation costs non-uniformly, i.e., more powerful devices have to bear higher costs for computation, communication and memory. Imaginable attacks in this scenario are that principals may cheat on the performance of their devices in order to save own resources at the expense of those devices that are less powerful, e.g. a kind of a DoS attack. In order to resist such attacks [6] introduces a new security requirement, called performance honesty. However, the protocol in [6] does not provide this requirement and is therefore vulnerable to this kind of attacks.

* Both authors were supported by the European Commission through IST-2002-507932 ECRYPT.
In this paper we propose a system model for mobile devices based on Trusted Computing (TCG specification [8]) which can be used to enforce the performance honesty in [6]. The idea is to distribute protocol operations within a device between trusted and non-trusted components. Note that as for PCs it is expected that in near future a wide range of mobile devices will be equipped with trusted hardware features.

\section{Related Work}

The solution in [6] assumes that every user is honest concerning his device' performance. Although [6] mentions two possible approaches against cheating, it does not provide any concrete realization.

The first approach is based on "incentives" and aims to discourage selfish behaviour of participants by making cooperation more attractive, e.g., [1] and [7]. Two flavours of such approaches have been introduced for packet forwarding: reputation-based and pricing-based schemes. In reputation-based schemes a misbehaving device looses its reputation that depends on the rate of the forwarded packets. Devices with lower reputation receive less services (punishment). In pricing-based schemes packet forwarding is treated as a service that may be priced, e.g., using virtual currency as in [2] or specific micro-payment systems as in [3]. Incentive-based approaches require high communication overhead to compute and update reputations of devices, or to handle the accounting. Besides that some pricing-based schemes still require tamper-resistant hardware components or virtual banks (trust authorities). Another problem with incentive-based approaches is that the performance can only be measured by the device itself (to the contrary, the packet forwarding ratio is measured by other devices during the communication with the challenged device).

Therefore, the second suggestion based on the trusted computing hardware, e.g., Trusted Platform Module (TPM) [8] seems more suitable and is also more efficient and effective.

\section{Model}

\subsection{Basic Definitions and Conventions}

We denote the set of devices (users) with $\mathcal{M}:=\left\{M_{1}, \ldots, M_{n}\right\}$. The performance ratio $\mu_{i}$ quantifies the performance capability of a mobile device $M_{i}$, 
and is the output of a benchmarking mapping that performs protocol specific network and cryptographic operations. A mobile device $M_{i}$ is considered to be more powerful than $M_{j}$ if $\mu_{i}>\mu_{j}$. A performance ratio order, denoted by $P$, of a mobile ad-hoc group is a sorted list of devices such that $\mu_{i} \geq \mu_{i+1}$ holds for any $M_{i}, M_{i+1}$ with $1 \leq i<n$. A signature scheme is denoted by a tuple (Sign, Verify) (the key generation algorithm is omitted). With $\sigma \leftarrow \operatorname{Sign}_{S K_{X}}(m)$ we denote the signature of a party $X$ on a message $m$ using the signing key $S K_{X}$. The verification algorithm ind $\leftarrow \operatorname{Verify}_{P K_{X}}(\sigma)$ returns ind $\in\{$ true, false $\}$. By Hash() we denote a cryptographic hash function. The deployed cryptographic primitives are assumed to be secure.

\subsection{Requirements}

The underlying security protocol should fulfill the specified security requirements stated for that protocol and the performance honesty requirement, i.e., that no principal is able to cheat on the performance ratio of its device. The following protocol is considered as a use case for our system model.

\section{Short Review of $\mu$ STR-H Protocols}

$\mu \mathrm{STR}-\mathrm{H}[6]$ is a contributory group key agreement protocol based on [4]. It computes the group key as a function of public contributions of all participants. $\mu$ STR-H consists of five main protocols: setup, join, leave, merge, and partition, that can be used to initialize the entire group and to handle dynamic events, respectively. These protocols provide semantic security against passive adversaries, i.e. it is computationally infeasible to distinguish between bits of the group key computed by $\mu \mathrm{STR}-\mathrm{H}$ and random bits. Additionally, assuming that all participants do not cheat on the performance of their devices $\mu$ STR-H protocols achieve cost fairness in heterogeneous environment, i.e., protocol costs are distributed between mobile devices according to their performance ratios.

$\mu$ STR-H protocols assume a public, reliable and authenticated broadcast channel within the group. Reliability can be achieved, for example, using reliable multicast protocols for ad-hoc networks, like RDG [5]. Authentication can be achieved using public key certificates, i.e., every participant $M_{i}$ has its own private/public key pair $\left(\right.$ skey $_{i}$, pkey $\left._{i}\right)$ which is used for message authentication. The public key pkey $_{i}$ should be certified. In the following description we omit the indication of the authentication procedure, i.e., of signature generation and verification.

$\mu$ STR-H sorts all group members (devices) according to the performance ratio order $P$. For a better efficiency all operations in $\mu$ STR-H are done in a cyclic group $\langle G>$ of prime order $t$ of points of an elliptic curve $E$ over a prime or binary finite field $\mathbb{F}_{q}$. Every group member $M_{i}$ has its own secret session random $r_{i} \in_{\mathcal{R}}\{1, \ldots, t-1\}$. The corresponding blinded value $R_{i}=r_{i} G$ (scalar-point multiplication equivalent to modular exponentiation in $\mathbb{Z}_{q}^{*}$ ) is public. Every $M_{i}$ saves a list $\mathbf{R}_{i}=\left\{R_{i}, \ldots, R_{n}\right\}$. During the protocol every member $M_{i}$ computes a set $\left\{k_{i}, \ldots, k_{n}\right\}$ where $k_{n}$ is the desired shared group key. For each $k_{i}$ there exists a public key $K_{i}=k_{i} G$. Note that $k_{1}=r_{1}$ and $K_{1}=R_{1}$. Each secret key $k_{i}(i>1)$ can be computed in two different ways: $k_{i}=\operatorname{map}\left(r_{i} K_{i-1}\right)$ or $k_{i}=\operatorname{map}\left(k_{i-1} R_{i}\right)$ where map is a point-to-integer mapping function. Every member $M_{i}$ computes and saves a list $\mathbf{k}_{i}=\left\{k_{i}, \ldots, k_{n}\right\}$ (note $M_{1}$ saves $\left\{k_{2}, \ldots, k_{n}\right\}$ ). In order to handle dynamic events every member $M_{i}(i>1)$ has also to save $K_{i-1}$.

Initialization: Figure 1 provides a full description of the setup protocol of $\mu$ STR-H based on the above preliminaries. For simplicity $i$ corresponds to member's $M_{i}$ position in $P$, and $\mu_{i}$ can be obtained from $P$.

$\begin{array}{ll}\text { - } & M_{i} \text { selects } r_{i} \text {, computes } R_{i} \text {, and broadcasts }\left(R_{i}, \mu_{i}\right) \\ \text { - } & M_{i} \text { computes } P=\left(M_{1}, \ldots, M_{n}\right) \text {, finds own index } i \text {, and } \\ & \text { saves } \mathbf{R}_{i}=\left(R_{i}, \ldots, R_{n}\right) \text {. Additionally, } M_{1} \text { computes } \mathbf{k}_{1}= \\ & \left(k_{2}, \ldots, k_{n}\right) \text {, and broadcasts }\left(K_{2}, \ldots, K_{n-1}\right) \text {. } \\ \text { - } & M_{i}(i>1) \text { computes } \mathbf{k}_{i}=\left(k_{i}, \ldots, k_{n}\right) \text {, and saves } K_{i-1} \text {. }\end{array}$

Figure 1. $\mu$ STR-H Setup

Due to the cost fairness requirement, less powerful devices have to compute less secret keys and save less blinded session randoms than more powerful devices that are located in the head of $P$. Figure 2 shows an example of a group of 5 members. Member $M_{3}$ uses its session random $r_{3}$, public key $K_{2}$ and blinded session randoms $R_{4}, R_{5}$ to compute secret keys $k_{3}, k_{4}$, and the group key $k_{5}$.

\begin{tabular}{ccccc} 
& $K_{2}$ & $K_{3}$ & $K_{4}$ & \\
& $k_{2}$ & $k_{3}$ & $k_{4}$ & $k_{5}$ \\
$R_{1}$ & $R_{2}$ & $R_{3}$ & $R_{4}$ & $R_{5}$ \\
$r_{1}$ & $r_{2}$ & $r_{3}$ & $r_{4}$ & $r_{5}$ \\
\hline$M_{1}$ & $M_{2}$ & $M_{3}$ & $M_{4}$ & $M_{5}$
\end{tabular}

Figure 2. Example of $\mu$ STR-H

Dynamic Events: A sponsor is used in $\mu \mathrm{STR}-\mathrm{H}$ to handle dynamic events. The sponsor $M_{s}$ is temporarily chosen from the group according to the actual state of $P . M_{s}$ changes own session random $r_{s}$ to achieve the freshness of the updated group key and broadcasts public keys that are required by other members to update the group key. There always exists at least one member who can verify information sent by the sponsor. In case of join the new member is inserted in $P$. The sponsor of join is the highestnumbered member below the position $j$ of the new member. If $j=1$ then the sponsor is $M_{2}$. In case of leave the leaving member is excluded from $P$. The sponsor of leave is the highest-numbered member below the position $d$ of the leaving member. If $d=1$ then the sponsor is $M_{2}$. For more details on $\mu$ STR-H protocols for join, leave, merge and partition, as well as its security analysis we refer to [6].

\subsection{Attacks against Perfomance Honesty}

In the following we consider attack scenarios against performance honesty in $\mu \mathrm{STR}-\mathrm{H}$. Members trying to cheat on performance ratios of their devices are called lying members. 
Individual Attacks: The adversary $M_{i}$ pretends that its device has a lower $\mu_{i}$ than it really does in order to get a better position in $P$ and save own costs during the protocol. Note that pretending to have a higher value for $\mu_{i}$ makes no sense, since it comes at adversary's expense. Another thinkable variation is that $M_{i}$ has several mobile devices with different performance ratios. $M_{i}$ 's goal is to use a higher-performance device for the protocol computations but obtain a better position in $P$ by submitting the performance ratio of its lower-performance device.

Collusion Attacks: Collusion may consist of different lying members so that at least one of them is able to obtain an advantageous position in $P$. It is also imaginable that some members of the collusion submit correct performance ratio parameters whereas other members cheat. In case of additive dynamic events (e.g., join) current members may collude in order to place new members on disadvantageous positions in $P$.

We require from $\mu$ STR-H to be resistant against all kinds of individual and collusion attacks.

\section{Trusted Components}

In this section, we propose an abstract system model based on TCG's (Trusted Computing Group) specifications ([9], [8]) that provide a practical solution to the missing link between the properties offered by a device platform and its configuration. Note that our system model uses trusted computing functionalities as black box.

Figure 3 illustrates this abstract model for a device $M$.

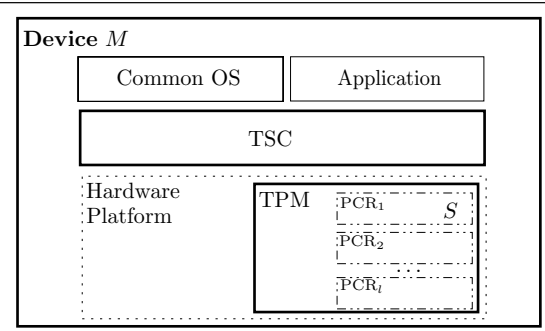

Figure 3. Abstract Model of $M$

The Trusted Computing Base (TCB) of a device consists of a Trusted Platform Module (TPM) and Trusted Software Component (TSC) described in the following.

Trusted Platform Module (TPM): The TPM provides a secure random number generator, non-volatile tamper-resistant storage, key generation algorithms, several cryptographic functions, amongst others a cryptographic hash function. A set of Platform Configuration Registers (PCR) are used in TPM to store hash values. The value of a PCR can only be modified as follows: $P C R_{i+1} \leftarrow \operatorname{Hash}\left(P C R_{i} \mid I\right)$, with the old value $P C R_{i}$, the new value $P C R_{i+1}$, and the input $I$ (e.g., a SHA-1 hash value). This process is called extending a PCR. The main task of the TPM is to bind relevant secrets used in the protocol to the properties of the device. For this purpose, the following set of the TCG security mechanisms can be applied.
Integrity Measurement and Platform Configuration: Integrity measurement is done during the boot process by computing a hash value of the initial platform state. For this purpose TPM computes a hash of (measures) the code and parameters of the BIOS and extends the first PCR register by this result. A chain of trust can be established if an enhanced BIOS and bootloader also measure the code they are transferring control to as next, e.g., to the operating system (OS). The security of the chain relies strongly on explicit security assumptions about the TPM. Thus, the PCR values $P C R_{0}, \ldots, P C R_{l}$ provide evidence of the system's state after boot. We call this state the platform's configuration, denoted by $S:=\left(P C R_{0}, \ldots, P C R_{l}\right)$.

Attestation: The TCG attestation protocol is used to give assurance about the platform configuration $S_{i}$ to a remote party. To guarantee integrity and freshness, this value and a fresh nonce provided by the remote party are digitally signed with an asymmetric key pair $\left(S K_{A I K}, P K_{A I K}\right)$ called Attestation Identity Key (AIK) that is under the sole control of the TPM.

Sealing: Data $D$ can be cryptographically bound to a certain platform configuration $S_{i}$ using an asymmetric key pair $(S K, P K)$ generated by the TPM. By sealing $D$ under $S$ we mean the encryption of $D$ using $P K$ and $S$. The unseal command releases the decrypted data $D$ only if for the current configuration $S^{\prime}$ holds $S^{\prime}=S$.

Trusted Software Component (TSC): The TSC includes elementary properties of a security kernel such as protecting its memory from being accessed and manipulated by unauthorized processes/programs and isolation of processes (e.g., common OS is isolated from the application). Since TSC is a software component it can be used to process operations that are too expensive for the TPM.

In our system model the operating system (OS) of the device and the application software such as $\mu$ STR-H protocol run on top of the TSC. Note that OS and the application software are not trusted components.

\section{TCG Meets $\mu$ STR-H}

\subsection{A Concrete System Model}

Based on the abstract model in Section 5 we propose a concrete model of a device in the context of the $\mu$ STR$\mathrm{H}$ protocols in Figure 4. Each device $M_{i}$ is the host of a

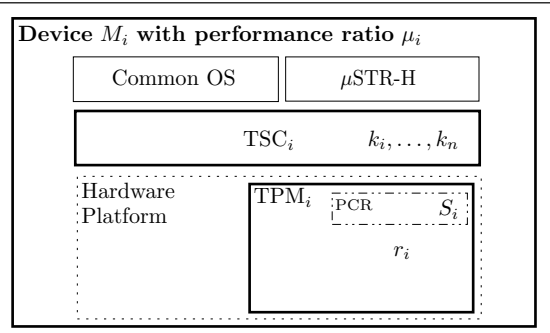

Figure 4. A Concrete System Model of $M_{i}$

$\mathrm{TPM}_{i} . \mathrm{TPM}_{i}$ is supposed to bind the secret session random $r_{i}$ to the performance ratio $\mu_{i}$ using the sealing function. Hence, the host can only access $r_{i}$ if $\mu_{i}$ is valid, i.e., if $M_{i}$ generates $r_{i}$ using one device he cannot use $r_{i}$ for the 
computations on another device. The computation of secret keys $k_{j}, i \leq j \leq n$ and corresponding public keys $K_{j}, i \leq j \leq n-1$ are performed by $\mathrm{TSC}_{i}$. However, $\mathrm{TSC}_{i}$ should not be able to perform certain security critical actions without involving the $\mathrm{TPM}_{i}$, or simply break the underlying security protocol by leaking the secret values $k_{i}$. Since $\mathrm{TSC}_{i}$ is a part of TCB, its measurement $S_{i}$ (e.g., a hash of $\mathrm{TSC}_{i}$ 's code which is computed at the boot time) will be included in the system configuration stored in PCRs, and hence the sealing functionality can be used to bind the secrets also to $S_{i}$. Since TCB represents a "constant" part of the device, any attempt to manipulate it by patches changes PCR values making unsealing impossible.

Note that the trust assumption on $\mathrm{TSC}_{i}$ concerns only software attacks and not attacks that attempt to read out the memory content by using hardware devices. The untrusted component of $\mu \mathrm{STR}-\mathrm{H}$ which runs on top of $\mathrm{TSC}_{i}$ and is isolated from other processes is responsible for the communication with other devices and performs operations which do not explicitly require the knowledge of device's secrets, e.g., verification of received signatures.

\subsection{Modified Protocols}

Every $\mathrm{TPM}_{i}$ chooses $r_{i} \in_{\mathcal{R}}\{1, \ldots, t-1\}$ and computes $R_{i}=r_{i} G$. To enforce performance honesty $\mathrm{TPM}_{i}$ seals $r_{i}$ under $\mu_{i}$ and the measurement $S_{i}$ of $\mathrm{TSC}_{i}$. TPM computes also the signature $\sigma_{i} \leftarrow \operatorname{Sign}_{S K_{A I K_{i}}}\left(R_{i}, \mu_{i}\right)$ testifying that $R_{i}$ is generated in a way that it is bound to $\mu_{i}$ and $S_{i}$. Signatures of $\mathrm{TPM}_{i}$ can be verified Verify $_{\mathrm{PK}_{A I K_{i}}}\left(R_{i}, \mu_{i}, \sigma_{i}\right)$ using its certificate $\operatorname{Cert}_{\mathrm{TPM}_{i}}$ that confirms the validity of $\mathrm{TPM}_{i}$ and the validity of its AIK, and is attached to the message. Note that $\operatorname{Cert}_{\mathrm{TPM}_{i}}$ can be used instead of public key certificates of users, i.e., device authentication instead of member authentication. $r_{i}$ remains inside $\mathrm{TPM}_{i}$, and is used (unsealed) by $\mathrm{TPM}_{i}$ only if $\mu_{i}$ and $S_{i}$ are correct. The user $M_{i}$ (untrusted $\mu \mathrm{STR}-\mathrm{H}$ component on top of $\mathrm{TSC}_{i}$ ) gains access to $R_{i}, \mu_{i}$ and $\sigma_{i}$.

Given $K_{i-1} \mathrm{TSC}_{i}$ computes $k_{i}=\operatorname{map}\left(r_{i} K_{i-1}\right)$ and $\left(k_{i+1}, \ldots, k_{n}\right)$ and $\left(K_{i}, \ldots, K_{n-1}\right)$ after it obtains the value $r_{i} K_{i-1}$ from $\mathrm{TPM}_{i} . M_{i}$ gains access to the public keys while the secret keys (including $k_{n}$ ) remain in the memory of $\mathrm{TSC}_{i}$. This is an important observation for the performance honesty. For simplicity we omit explicit notation of the interaction between $M_{i}, \mathrm{TPM}_{i}$, and $\mathrm{TSC}_{i}$ according to Figure 4 in the following description of our protocols assuming that required data is transmitted over defined interfaces. Further, we assume that it is possible to obtain $\mu_{i}, \sigma_{i}$ and $\operatorname{Cert}_{\mathrm{TPM}_{i}}$ using $i$ and $P$.

If in $\mu$ STR-H a sponsor $M_{s}$ has to change $r_{s}$ then $\mathrm{TPM}_{s}$ chooses a new value for $r_{s}$, computes $R_{s}$, seals $r_{s}$ under the configuration $\left(\mu_{s}, S_{s}\right)$ and generates a signature on $R_{s}, \mu_{s}$ using AIK.

$\mu$ STR-H Setup: Let $M_{1}, \ldots, M_{n}$ be participants wishing to initialize a mobile ad-hoc group.

- $\mathrm{TPM}_{i}$ selects $r_{i}$, computes $R_{i}$ and $\sigma_{i} . M_{i}$ broadcasts $\left(R_{i}, \mu_{i}, \sigma_{i}\right.$, Cert $\left._{\mathrm{TPM}_{i}}\right)$.

- $M_{i}$ verifies $\sigma_{j}$ for all $1 \leq j \leq n$, computes $P$, and stores $\mathbf{R}_{i}$.
Additionally: $\mathrm{TSC}_{1}$ computes $\mathbf{k}_{1}=\left(k_{2}, \ldots, k_{n}\right)$, $\left(K_{2}, \ldots, K_{n-1}\right) . M_{1}$ broadcasts $\left(K_{2}, \ldots, K_{n-1}\right)$.

- For all $i>1: M_{i}$ stores $K_{i-1}$. $\mathrm{TSC}_{i}$ computes $\mathbf{k}_{i}=$ $\left(k_{i}, \ldots, k_{n}\right)$.

Note that after the completion of the protocol the performance ratio order $P$ contains only devices with verifiable TPM's signatures.

$\mu$ STR-H Join: A new member $M_{j}$ is inserted in $P$ according to its performance ratio $\mu_{j}$. The new group key is denoted $k_{n+1}$.

- New member's $\mathrm{TPM}_{j}$ selects $r_{j}$, computes $R_{j}$ and $\sigma_{j} . M_{j}$ broadcasts $\left(R_{j}, \mu_{j}, \sigma_{j}, \operatorname{Cert}_{\mathrm{TPM}_{j}}\right)$.

- After successful verification of $\sigma_{j}$ every $M_{i}$ inserts $M_{j}$ in $P$, renumbers all $M_{i}(i>j)$ to $M_{i+1}$, and adds $R_{j}$ to $\mathbf{R}_{i}$. (note if verification of $\sigma_{j}$ fails then the protocol stops).

Additionally: $\mathrm{TPM}_{s}$ selects new $r_{s}$, computes $R_{s}$ and $\sigma_{s}$. $\mathrm{TSC}_{s}$ recomputes $\mathbf{k}_{s}=\left(k_{s}, \ldots, k_{n+1}\right)$ and $\left(K_{s}, \ldots, K_{n}\right)$. $M_{s}$ broadcasts $\left(P, \quad \sigma_{s}, \quad R_{s}, \quad\left(R_{j+1}, \ldots, R_{n+1}\right)\right.$, $\left.\left(K_{s}, \ldots, K_{n}\right), \operatorname{Cert}_{\mathrm{TPM}_{s}}\right)$.

- $M_{j}$ after successful verification of $\sigma_{i}$ for all $1 \leq i \leq n+1$ stores $P$, finds own index $j$, stores $K_{s}$ and $\mathbf{R}_{j}=\left(R_{j}, \ldots, R_{n+1}\right)$. TSC ${ }_{j}$ computes $\mathbf{k}_{j}=\left(k_{j}, \ldots, k_{n+1}\right)$. For all $i<s$ : After successful verification of $\sigma_{s} M_{i}$ updates $R_{s}$ in $\mathbf{R}_{i}$. $\operatorname{TSC}_{i}$ recomputes $\left(k_{s}, \ldots, k_{n+1}\right)$ in $\mathbf{k}_{i}$.

For all $i>s$ : $M_{i}$ updates $K_{i-1}$. $\mathrm{TSC}_{i}$ recomputes $\left(k_{i}, \ldots, k_{n+1}\right)$.

$\mu$ STR-H Leave: A leaving member $M_{d}$ is removed from $P$. The new group key is denoted $k_{n-1}$.

- $M_{i}$ deletes $M_{d}$ from $P$, if $i<d$ also $R_{d}$ from $\mathbf{R}_{i}$, and $k_{d}$ from $\mathbf{k}_{i}$, and renumbers all $M_{i}(i>d)$ to $M_{i-1}$.

Additionally: $\mathrm{TPM}_{s}$ selects new $r_{s}$, computes $R_{s}$ and $\sigma_{s}$. $\mathrm{TSC}_{s}$ recomputes $\mathbf{k}_{s}=\left(k_{s}, \ldots, k_{n-1}\right)$ and $\left(K_{s}, \ldots, K_{n-2}\right)$. $M_{s}$ broadcasts $\left(R_{s}, \sigma_{s}, \operatorname{Cert}_{\mathrm{TPM}_{s}},\left(K_{s}, \ldots, K_{n-2}\right)\right.$.

- For all $i<s: M_{i}$ after successful verification of $\sigma_{s}$ updates $R_{s}$ in $\mathbf{R}_{i} . \mathrm{TSC}_{i}$ recomputes $\left(k_{s}, \ldots, k_{n-1}\right)$ in $\mathbf{k}_{i}$.

For all $i>s: M_{i}$ updates $K_{i-1}$. $\mathrm{TSC}_{i}$ recomputes $\mathbf{k}_{i}=$ $\left(k_{i}, \ldots, k_{n-1}\right)$.

$\mu$ STR-H Merge: Two groups, $\mathrm{G}^{\prime}$ of size $n^{\prime}$ and $\mathrm{G}^{\prime \prime}$ of size $n^{\prime \prime}$, are merging to a common group G. The resulting $P$ is computed by merging $P^{\prime}$ and $P^{\prime \prime}$. The new group key is denoted $k_{n^{\prime}+n^{\prime \prime}}$.

- $M_{1}^{\prime}$ and $M_{1}^{\prime \prime}$ broadcast $\left(P^{\prime},\left(R_{1}^{\prime}, \ldots, R_{n^{\prime}}^{\prime}\right)\right)$ and $\left(P^{\prime \prime}\right.$, $\left.\left(R_{1}^{\prime \prime}, \ldots, R_{n^{\prime \prime}}^{\prime \prime}\right)\right)$, respectively.

- Every member $M_{i}$ verifies signatures $\sigma_{j}$ for all $1 \leq j \leq n^{\prime}+n^{\prime \prime}$, merges $P^{\prime}$ and $P^{\prime \prime}$ to $P$, and stores $\mathbf{R}_{i}=\left(R_{i}, \ldots, R_{n^{\prime}+n^{\prime \prime}}\right)$.

Additionally: $\mathrm{TPM}_{s}$ selects new $r_{s}$, computes $R_{s}$ and $\sigma_{s} . \mathrm{TSC}_{s}$ recomputes $\mathbf{k}_{s}=\left(k_{s}, \ldots, k_{n^{\prime}+n^{\prime \prime}}\right)$ and $\left(K_{s}, \ldots, K_{n^{\prime}+n^{\prime \prime}-1}\right) . \quad M_{s}$ broadcasts $\left(R_{s}, \sigma_{s}\right.$, $\left.\left(K_{s}, \ldots, K_{n^{\prime}+n^{\prime \prime}-1}\right)\right)$.

- For all $i<s: M_{i}$ after successful verification of $\sigma_{s}$ updates $R_{s}$ in $\mathbf{R}_{i}$. TSC $i$ recomputes $\left(k_{s}, \ldots, k_{n^{\prime}+n^{\prime \prime}}\right)$ in $\mathbf{k}_{i}$.

For all $i>s$ : $M_{i}$ updates $K_{i-1}$. $\mathrm{TSC}_{i}$ recomputes $\mathbf{k}_{i}=$ $\left(k_{i}, \ldots, k_{n^{\prime}+n^{\prime \prime}}\right)$. 
$\mu$ STR-H Partition: A subgroup $\mathrm{G}^{\prime}$ of size $v$ leaves group $\mathrm{G}$ of size $n$. The new group key is denoted $k_{n-v}$.

- $M_{i}$ deletes all $M_{j}^{\prime}$ from $P$, if $i<j$ also $R_{j}^{\prime}$ from $\mathbf{R}_{i}$ and $k_{j}^{\prime}$ from $\mathbf{k}_{i}$, and renumbers all survived members $M_{i}$ accordingly. Additionally: $\mathrm{TPM}_{s}$ selects new $r_{s}$, computes $R_{s}$ and $\sigma_{s}$. TSC $\mathrm{TS}_{s}$ recomputes $\mathbf{k}_{s}=\left(k_{s}, \ldots, k_{n-v}\right)$ and $\left(K_{s}, \ldots, K_{n-v-1}\right) . M_{s}$ broadcasts $\left(R_{s},\left(K_{s}, \ldots, K_{n-v-1}\right)\right)$.

- For all $i<s: M_{i}$ after successful verification of $\sigma_{s}$ updates $R_{s}$ in $\mathbf{R}_{i} . \mathrm{TSC}_{i}$ recomputes $\left(k_{s}, \ldots, k_{n-v}\right)$ in $\mathbf{k}_{i}$.

For all $i>s: M_{i}$ updates $K_{i-1}$. $\mathrm{TSC}_{i}$ recomputes $\mathbf{k}_{i}=$ $\left(k_{i}, \ldots, k_{n-v}\right)$.

\subsection{Security Analysis}

In this section we informally analyse the performance honesty of our modifications to $\mu \mathrm{STR}-\mathrm{H}$. Note that other security issues of $\mu \mathrm{STR}-\mathrm{H}$ are still valid since our modifications concern only internal computations within the device.

In the modified protocols every $M_{i}$ is able to compute $P$ and check own position in $P$ by verifying the TPMs' signatures $\sigma_{j}, 1 \leq j \leq n$ of other devices. $\sigma_{i}$ ensures that $R_{i}$ has been generated within the device those performance ratio is $\mu_{i}$. Since $\mathrm{TPM}_{i}$ is trusted $r_{i}$ never leaves its memory. The secret keys $\mathbf{k}_{i}=\left(k_{i}, \ldots, k_{n}\right)$ are computed by $\mathrm{TSC}_{i}$. Since $k_{i}=\operatorname{map}\left(r_{i} K_{i-1}\right)$ and $\mathrm{TSC}_{i}$ does not know $r_{i} \mathrm{TPM}_{i}$ must be involved in the computation. Since $\mathrm{TSC}_{i}$ is trusted secret keys $\mathbf{k}_{i}=\left(k_{i}, \ldots, k_{n}\right)$ never leave its memory. Also, $r_{i}$ is bound to $\mathrm{TSC}_{i}$, i.e., any adversarial modification of $\mathrm{TSC}_{i}$ would make unsealing impossible. Hence, $M_{i}$ does not get any direct access to the group key $k_{n}$ or to any other secrets that needed to compute it. Therefore, $M_{i}$ must use the same device throughout the protocol and ask $\mathrm{TSC}_{i}$ to perform application specific operations using $k_{n}$. Since the signature scheme is assumed to be secure $M_{i}$ cannot forge $\sigma_{i}$ and cheat on $\mu_{i}$. Thus, modifed $\mu$ STR-H provides performance-honesty. For completeness, we consider the security against individual and collusion attacks from Section 4.1.

Security against individual attacks: User $M_{i}$ cannot lie on its performance ratio $\mu_{i}$ because $\mu_{i}$ and $R_{i}$ are signed by $\mathrm{TPM}_{i}$. Since the signature scheme is secure $M_{i}$ cannot forge $\sigma_{i}$ for another value of $\mu_{i}$. If $M_{i}$ submits a signature that cannot be verified by other users then $M_{i}$ 's contribution $R_{i}$ is dropped and $M_{i}$ cannot compute the group key. If $M_{i}$ replays a valid signature $\sigma_{i}$ then it is assigned to a position in $P$, but cannot compute the group key because it does not know $r_{i}$. Similarly, if $M_{i}$ submits $\sigma_{i}$ of its lower-performance device but wishes to use its higherperformance device to compute the group key it fails because $r_{i}$ remains inside $\mathrm{TPM}_{i}$ of the lower-performance device.

Security against collusion attacks: Consider a collusion $\mathcal{C}=\left\{M_{i_{1}}, \ldots, M_{i_{k}}\right\}$. Assume that all members in $\mathcal{C}$ lie on their performance ratios. Since they cannot forge TPMs' signatures there should be at least one honest member $M_{j} \notin \mathcal{C}$. $M_{j}$ is able to identify all lying members, because $M_{j}$ verifies the order of $P$ by proving the TPMs' sig- natures. Assume that all members in $\mathcal{C}$ submit correct performance ratios. Let $\mathcal{H} \subset \mathcal{C}$ be a set of collusion members who wish to save own costs and obtain the group key from members in $\mathcal{C}-\mathcal{H}$. As described above $r_{i}$ remains inside $\mathrm{TPM}_{i}$ and all $\mathbf{k}_{i}=\left(k_{i}, \ldots, k_{n}\right)$ in the memory of $\mathrm{TSC}_{i}$. Therefore, even if members in $\mathcal{C}-\mathcal{H}$ compute $k_{n}$ they do not get any direct access to it or to any other secret value (e.g., $r_{i}$ or $\mathbf{k}_{i}$ ). Hence, they cannot provide members in $\mathcal{H}$ with the required secret information. Assume that during the communication session all group members collude in order to assign joining members to disadvantageous positions in $P$. Every new member checks own position in the updated $P$ by verifying the TPMs' signatures of all devices, and is, therefore, able to identify all lying members.

\section{Conclusion}

In this paper we have considered the deployment of trusted computing technology to control the access of mobile devices to the input/output of a security protocol depending on the devices' properties. In the context of secure group communication ( $\mu$ STR-H protocol) we have designed a system model for mobile devices to achieve this control considering the performance of every involved mobile device as a property.

\section{References}

[1] S. Basagni, M. Conti, S. Giordano, and I. Stojmenović, editors. Mobile Ad Hoc Networking. Wiley-IEEE Press, 2004.

[2] L. Buttyan and J.-P. Hubaux. Enforcing service availability in mobile ad-hoc wans. In MobiHoc '00: Proceedings of the 1st ACM international symposium on Mobile ad hoc networking \& computing, pages 87-96, Piscataway, NJ, USA, 2000. IEEE Press.

[3] M. Jakobsson, J.-P. Hubaux, and L. Buttyan. A micropayment scheme encouraging collaboration in multi-hop cellular networks. In Financial Cryptography 2003, volume 2742 of Lecture Notes in Computer Science, pages 15-33. Berlin: Springer-Verlag, 2003.

[4] Y. Kim, A. Perrig, and G. Tsudik. Communication-efficient group key agreement. In Information Systems Security, Proc. of the 17th International Information Security Conference, IFIP SEC'01, 2001.

[5] J. Luo, P. T. Eugster, and J.-P. Hubaux. Route driven gossip: Probabilistic reliable multicast in ad hoc networks. In INFOCOM, 2003.

[6] M. Manulis. Key Agreement for Heterogeneous Mobile AdHoc Groups. In Proceedings of 11th International Conference on Parallel and Distributed Systems Volume 2 International Workshop on Security in Networks and Distributed Systems, pages 290-294. IEEE Computer Society, 2005.

[7] P. Michiardi and R. Molva. A game theoretical approach to evaluate cooperation enforcement mechanisms in mobile ad hoc networks. In Proceedings of Modeling and Optimization in Mobile, Ad Hoc, and Wireless Networks (WiOpt'03), 2003.

[8] Trusted Computing Group (TCG). TPM main specification, Version 1.2, http://www. trustedcomputinggroup. org, November 2003.

[9] Trusted Computing Platform Alliance (TCPA). TCPA main specification, Version 1.1.b, February 2002. 